Relations industrielles

Industrial Relations

\title{
Le rôle politique des syndicats aux États-Unis
}

Les syndicats dans la vie américaine

\section{Leon A. Dale}

Volume 17, numéro 4, octobre 1962

URI : https://id.erudit.org/iderudit/1021477ar

DOI : https://doi.org/10.7202/1021477ar

Aller au sommaire du numéro

\section{Éditeur(s)}

Département des relations industrielles de l’Université Laval

\section{ISSN}

0034-379X (imprimé)

1703-8138 (numérique)

Découvrir la revue

\section{Citer cet article}

Dale, L. A. (1962). Le rôle politique des syndicats aux États-Unis : les syndicats dans la vie américaine. Relations industrielles / Industrial Relations, 17(4),

422-443. https://doi.org/10.7202/1021477ar

Tous droits réservés @ Département des relations industrielles de l’Université Laval, 1963
Ce document est protégé par la loi sur le droit d'auteur. L’utilisation des services d'Érudit (y compris la reproduction) est assujettie à sa politique d'utilisation que vous pouvez consulter en ligne.

https://apropos.erudit.org/fr/usagers/politique-dutilisation/ 


\title{
Le rôle politique des syndicats aux Etats-Unis
}

\author{
Les syndicats dans la vie américaine
}

Leon A. Dale

a) LE MOUVEMENT SYNDICAL DANS L'ARÈNE POLITIQUe

La position des syndicats au sein de la société américaine a subi de profonds changements, particulièrement depuis 1935. Le nombre des syndiqués a quadruplé passant de $3,800,000$ en 1935 à $15,600,000$ en 1948. ${ }^{1}$ Etant donné que ni le chiffre de la population ni celui de la main-d'oeuvre n'a quadruplé ni même doublé, il était inévitable que l'influence respective des syndicats se soit accrue considérablement. Le rapport des forces en présence a donc changé; les raisons de ce changement expliquent en partie la situation présente.

A la fin de la deuxième guerre mondiale, les syndicats se trouvaient dans une bien meilleure position qu'au début. Leurs réserves financières ainsi que le nombre de leurs adhérents s'étaient considérablement accrus. A cette époque, en dépit de cette amélioration, on n'avait pas une perspective optimiste de l'avenir syndical. Car à la fin des hostilités, de nombreux économistes avaient prédit une dépression économique d'importance accompagnée d'un nombre élevé de chômeurs alors que le gouvernement allait annuler ses contrats de guerre. Cette crainte d'une dépression économique, jointe à celle d'une campagne patronale contre le système des conventions collectives, avait incité les syndicats à prendre l'offensive. Cette campagne syndicale eut deux aspects : 1) un recrutement intensif destiné à augmenter les effectifs syndicaux, particulièrement dans le sud des Etats-Unis, et 2) une pression sur les patrons, ostensiblement pour augmenter les salaires, mais aussi pour protéger et garantir la sécurité des syndicats dans les usines. Cette campagne acquit d'autant plus de vigueur que la dépression envisagée ne se produisit pas. Cependant, dans une certaine mesure, le pessimisme syndical (et l'offensive qui en résulta) eut pour conséquence le vote de la loi Taft-Hartley. De nombreux aspects de cette loi représentent une véritable métarmorphose qui s'est accomplie dans l'attitude du gouvernement vis-à-vis des syndicats et vice-versa. Pour bien comprendre sa portée, il faut remonter à l'époque héroïque des syndicats.

A leurs débuts, les syndicats n'étaient pas. sujets à la juridiction du gouvernement fédéral mais à celle des différents états, et en gé-
DALE, Leon A., Chairman, Department of Industrial Relations, University of Bridgeport, Bridgeport, Connecticut.

(1) Monthly Labor Review, février 1949, p. 224. 
néral à l'hostilité caractérisée des tribunaux régionaux. Cette hostilité fondamentale qui fut de règle pendant tout le 19ème siècle, et même le premier tiers du 20ème, s'explique en majeure partie par la puissance du droit de la propriété privée. Le pays avait été colonisé à son origine par des pionniers qui créaient littéralement leur propriété. Ceci était vrai non seulement pour les fermiers, mais aussi pour les petites industries qui se développaient. La propriété privée devint une institution dotée de caractères sacrés. Or le syndicalisme représente une attaque contre la propriété privée en ce sens qu'il pose des restrictions au droit du patron à contrôler sa «propriété productrice ${ }^{2}{ }^{2}$. L'hostilité au syndicalisme fut fonction de la puissance du concept de la propriété privée. Ce concept avait acquis une force d'autant plus grande aux EtatsUnis que les immigrants européens fuyaient l'arbitraire du seigneur et la difficulté d'obtenir des terres «en toute propriété »sans être assujettis à certaines «corvées» ou obligations. Cela explique aussi en partie la force du concept de la liberté de l'individu, concept que l'on estimait froissé par les «conspirations $\rrbracket^{3}$ syndicales. Les syndciats constituaient une sorte de révolution pacifique par le fait même qu'ils se composaient de travailleurs agissant de concert pour protéger leurs intérêts. A une époque où l'accent se trouvait mis sur l'initiative individuelle, on les considérait coupables du délit de «conspiration». Quoique le délit de «conspiration» fut théoriquement abandonné en 1842 et la légalité des syndicats jugée par le critère des buts qu'ils poursuivaient, il n'en reste pas moins vrai que leur doctrine ne fut abandonnée qu'avec difficulté par les tribunaux américains à la fin du 19ème siècle. ${ }^{4}$

L'apparition et le développement du syndicalisme entraînaient une modification, en quelque sorte un «adoucissement» au principe de la propriété privée, et de fait entraînaient un changement dans le droit américain. Ce changement représentait la raison d'être des syndicats eux-mèmes, une réaction à la sévérité des conditions entraînées par la conception de la propriété privée. Mais ce changement ne pouvait s'effectuer qu'alors que la puissance des syndicats s'était déjà manifestée, c'est-à-dire lorsque ceux-ci avaient la possibilité d'engager les hostilités avec une chance de succès ou de sérieuses répercussions pour le public. Dès leur apparition, les activités syndicales eurent leur effet dans le domaine politique où elles furent âprement discutées. Dès leur apparition donc, les syndicats furent presqu'obligés de descendre dans l'arène politique. Préoccupés surtout par des résultats d'ordre pratique, les syndicats utilisaient soit la grève, soit une pression sur les Chambres de Représentants des divers états. Une activité politique de la part des syndicats leur était indispensable sous un régime judiciaire qui leur

(2) Cf. Selig Perlman, A Theory of the Labor Movement, Augustus M. Kelley, New York, 1949, particulièrement pp. 155-159.

(3) Appelées en France vers la même époque «coalitions ». Cf. aussi Relations Industrielles, Vol. 17 , no 3 , p. 305 .

(4) Cf. Charles O. Gregory, Labor and the Law, W.W. Norton and Co., Inc., New York; 1949, pp. 13-30 et particulièrement pp. 27-30 pour une discussion du cas célèbre de «Commonwealth vs Hunt》 de 1842 où la décision du tribunal fut pour la première fois basée sur la légalité des intentions des travailleurs. 
était hostile. D’autre part, certaines demandes syndicales ne pouvaient s'effectuer que par l'intermédiaire des corps législatifs, telles qu'un système obligatoire (et gratuit) d'instruction publique, l'indemnité de chômage, les assurances sociales, des cités ouvrières, des soins médicaux à des tarifs accessibles aux ouvriers, ainsi que des mesures financières en cas de dépression économique. Certains objectifs qui pouvaient être atteints par l'intermédiaire de conventions collectives l'étaient plus facilement à l'aide d'actes législatifs, tels que ceux concernant les salaires minima, un nombre maximum d'heures de travail par semaine et l'élimination de la main-d'oeuvre recrutée chez les enfants.

La première campagne politique entreprise par un syndicat eut lieu aux Etats-Unis en 1828 quand le syndicat des mécaniciens (Mechanics' Union of Trade Associations) constitua le «Parti des Travailleurs ». Le succès temporaire de ce premier parti ouvrier incita les travailleurs de vil'es autres que Philadelphie et telles que New-York, Boston, Albany, à former des partis politiques similaires. A l'aide de ces partis, la classe ouvrière pensait pouvoir changer les conditions économiques (une dépression économique était alors en cours), écourter la longueur des journées de travail et éliminer les délits de conspiration. Le manque d'expérience des ouvriers dans le domaine politique, et le fait que les deux autres partis politiques n'éprouvaient aucun scrupule à s'approprier les plate-formes électorales du parti ouvrier amenèrent leur déconfiture dans les cinq années qui suivirent. ${ }^{5}$ Après cela, la classe ouvrière en revint aux moyens de pression économique tels que la grève.

En 1866, «L'Union Nationale du Travail » (National Labor Union) fut constituée par des syndicalistes cherchant à unifier leurs forces alors éparpillées. Cette Union qui participait aux campagnes électorales prit une part active dans le mouvement pour la loi de huit heures et établit un bureau à Washington, la capitale, pour faire pression sur les membres du Congrès. L'Union Nationale du Travail désirait même établir un parti ouvrier indépendant, mais, pour pratiquement les mêmes raisons, elle rencontra le même échec que ses prédécesseurs. Il fallut attendre 1881, date de la formation de l'AFL, pour voir s'établir un mouvement indépendant du parti Démocrate et du parti Républicain, défavorables à un parti politique ouvrier. ${ }^{6}$

\section{b) L'ACTION POLITIQUe DE L'AFL}

- Gompers déclarait toujours que le travailleur devait s'intéresser à la politique et à la législation. Il voulait, cependant, empêcher les tra-

(5) Cf. Selig Perlman, A History of Trade Unionism, in the United States, Augustus N. Kelley, New York, p. 18.

(6) Cf. Relations Industrielles, Vol. 17, no 3, p. 309. 
vailleurs de devenir les disciples de n'importe quel parti politique quel que soit son nom. \$*

En dépit de sa position, l'AFL soutient officiellement la candidature de Victor Berger qui fut le premier représentant socialiste au Congrès des Etats-Unis. De plus, l'AFL vint toujours à l'aide des minorités, que ce soient les anarchistes, socialistes ou objecteurs de conscience et que ceux-ci soient ou non membres de l'AFL. Quoique l'AFL désirât syndiquer les travailleurs de couleur, certains de ses affiliés refusaient de les accepter. Dans ce cas, l'AFL leur offrait d'établir un syndicat qui serait affilié directement à l'AFL. Cette dernière réalisait que cela ne représentait pas une solution adéquate, mais préférait faire quelque chose de concret qui entretemps lui permettait de persuader ses affiliés de changer d'avis.

La Constitution de la Fédération Américaine du Travail déclarait qu'un de ses objectifs était «d'obtenir des lois qui soient dans l'intérêt des masses ouvrières ${ }^{7}$ et que :

* Le Conseil Exécutif aura le devoir de surveiller les mesures législatives concernant les intérêts des travailleurs et de commencer des démarches en vue de mesures législatives, chaque fois que cela sera nécessaire et à la discrétion du Congrès (de l'AFL) > 8 ;

quoique l'AFL fut indépendante des partis politiques, sa Constitution indiquait clairement que l'AFL non seulement n'était pas en dehors de la vie politique du pays, mais y prenait une part active. Cependant, la Constitution de l'AFL n'indiquait pas de quelle manière cette participation était envisagée.

Comme un spécialiste dans les questions intéressant le mouvement syndical l'a déclaré ${ }^{\circ}$, l'AFL utilisait dans une certaine mesure la technique des conventions collectives en matières po'itiques. Au moment des élections, l'AFL disposait d'un certain nombre de voix dans un territoire donné, ou du moins pensait disposer d'un certain nombre de voix, car on s'est aperçu que, dans de nombreux cas, les syndiqués ne votaient pas comme le suggéraient leurs leaders. De toute façon, l'AFL était en mesure de soutenir non pas un parti mais certains candidats qui, par raison de leurs «performances» passées, présentes ou «promises», semblaient favorables aux objectifs poursuivis par l'AFL.

(*) The A.F. of L. in the Time of Gompers, par Philip Taft, cite dans * Gompers Architect of American Labor », International Free Trade Unions, Vol. 12, no 12, décembre 1957, p. 6.

(7) Constitution of the American Federation of Labor (as adopted at the sixtyninth Convention held at Houston, Texas, September 18-23, inclusive, 1950), p. 3.

(8) Ibid. p. 23.

(9) Henry David, Labor Problems in America, E. Stein, J. Davis, and others, pp. 399-400, Farrar and Rinehart, Inc., New York, 1940. 
L'AFL «faisait de la politique» à trois échelons différents : celui de la ville, de l'état et du gouvernement fédéral.

A l'échelon du gouvernement fédéral, lors des élections présidentielIes (qui ont lieu tous les quatre ans), l'AFL établissait une liste de réformes qu'elle soumettait au parti Démocrate et au parti Républicain. Ceci fait, elle comparait les plateformes électorales des deux partis et établissait dans quelle mesure il était tenu compte de ses désirs. Puis elle communiquait à ses affiliés et à la presse le résultat de ses conciusions. Le bureau de l'AFL qui s'occupait plus spécialement de politique durant ces années d'élections présidentielles et qui s'appelait le "Comité Impartial de la Campagne Electorale» (Non-Partisan Political Campaign Committee) se composait du président de l'AFL et d'une partie des membres du Conseil Exécutif. La Fédération Américaine du Travail démontrait son influence de manière encore plus frappante au cours de l'élection des membres du Congrès fédéral ${ }^{10}$, élections qui ont lieu tous les deux ans. Fidè è la devise de Samuel Gompers, l'AFI, continuait sa tradition de «récompenser ses amis et de punir ses ennemis » (Reward your friends and punish your enemies) dans le domaine politique. Le Comité Impartial de l'AFL faisait une étude des candidats en présence et plus particulièrement de leur attitude envers les questions importantes intéressant les travailleurs (comme la loi Taft-Hartley ). Les résultats de ces études étaient alors diffusés aux adhérents de l'AFL pour que ceux-ci puissent voter en connaissance de cause.

A l'échelon de l'état et de la ville, la technique était sensiblement la même avec cette différence que c'étaient les fédérations d'état affiliées à l'AFL ou les conseils centraux des villes réunissant les syndicats locaux de l'AFL qui exécutaient ce travail et faisaient parvenir à leurs adhérents les renseignements nécessaires.

L'AFL possédait en outre un Comité Législatif (Legislative Committee) qui se composait de trois membres choisis par le Conseil Exécutif. Le Comité Législatif étudiait les propositions de loi et les projets de loi présentés au Congrès fédéral et faisait pression sur les membres du Congrès lors de la discussion d'actes législatifs intéressant l'AFL. ${ }^{11}$ Quelquefois, des membres de la Chambre des Représentants, ou des Sénateurs, qui étaient soit d'accord avec un des buts particuliers de l'AFL ou bien simplement conscients de la puissance électora'e de ce dernier, proposaient des projets de loi parrainés par le mouvement syndical. Un Comité de Conférence Législative (Legislative Conference Committee) se réunissait une fois par mois quand le Congrès était en séance. A l'instar du CIO et des syndicats indépendants importants, l'AFL cherchait aussi à influencer le corps législatif au moyen de «témoignages». Quand le comité de la Chambre des Représentants ou

(10) Tous les deux ans, la Chambre des Représentants est complètement renouvelée ainsi qu'un tiers du Sénat.

(11) En raison de l'ampleur des sujets qui intéressent le mouvement syndical, ce comité a un travail considérable; voir plus loin. 
du Sénat chargé de l'élaboration d'un texte de loi concernant de près ou de loin le mouvement syndical tient séance, des délégués syndicalistes donnent leur avis, témoignent», sur la législation proposée. Le poids de cet avis, ou «témoignage», dépend souvent de l'importance de l'organisation représentée par les «témoins». Souvent aussi les organisations intéressées envoient aux membres du Congrès des lettres ou circulaires détaillant 'eur position. L'AFL dépensait environ 52,000 dollars par an pour ses activités destinées à influencer le passage d'actes législatifs. ${ }^{12}$ Bref, les activités législatives du Congrès étaient surveillées de très près par l'AFL et le mouvement syndical en général qui, au moment de la discussion de projets de loi importants n'hésitaient pas à mobiliser leurs ressources soit pour chercher à contrecarrer le passage d'un projet de loi qu'ils jugaient inacceptable soit pour soutenir au contraire un texte de loi qu'ils considéraient comme favorable. Une telle activité appuyée par les moyens publicitaires modernes ne pouvait manquer de se faire sentir à longue ou courte échéance. Ce n'était pas par hasard que depuis 1897 le quartier général de l'AFL se trouvait situé à Washington, de même que celui du CIO et ceux d'environ trentecinq syndicats. A tel point que les organisations syndica'es possèdent plus de 15,000,000 de dollars en biens immobiliers à Washington. ${ }^{13}$

En 1924, pour la première fois au cours de son histoire, se départissant de sa réserve, l'AFL accorda officiellement son appui à La Follette qui posait sa candidature à la Présidence des Etats-Unis au nom du parti Progressiste. La Follette perdit l'éection. Rompant avec ses traditions pour la deuxième fois, en 1952, l'AFL donna officiellement son appui à Stevenson qui au nom du parti Démocrate se présentait contre Eisenhower. Stevenson perdit l'élection. Quelquefois, l'AFL jouait un rôle important aux Congrès des partis désignant leurs candidats à la Présidence. C'est ainsi qu'en 1944, plus de cinquante officiels de l'AFL assistaient au Congrès du parti Démocrate en tant que délégués de ce parti; ‘a nomination de Roosevelt à la Présidence et celle de Truman à la vice-présidence furent partiellement dues à leur influence.

En 1947, après le passage de la loi Taft-Hartley, l'AFL établit la Ligue du Travail pour l'Instruction Politique (Labor's League for Political Education). Le financement de cette nouvelle organisation était assuré par des contributions de la part des syndiqués. Quoique la loi Taft-Hartley interdise les contributions directes à une campagne électorale, l'AFL estimait qu'elle n'interdisait pas les versements destinés à un travail éducatif tel que des émissions radiophoniques commentant les nouvelles et un service d'information et d'éducation. ${ }^{14}$ Cependant les fonds recueillis de cette manière déçurent les espérances des militants. En 1950, moins de 600,000 dollars furent obtenus et en 1952

(12) AFL Convention Proceedings, 1952, p. 280.

(13) W.M. Kiplingen, Washington is Like That, Harper \& Brothers, New York, 1947 , p. 192 , cite $10,000,000$ de dollars. Mais en 10 ans non seulement la valeur des biens immobiliers a de par beaucoup augmenté mais aussi le nouveau quartier général de l'AFL-CIO a coûté entre 4 et 5 millions de dollars.

(14) Voir Relations Industrielles, Vol. 17, no 3, p. 320. 
seulement 252,000 dollars. George Meany, qui était alors président de l'AFL exprima l'espoir qu'en 1954 plus de 600,000 dollars représenteraient la contribution des $10,300,000$ membres $^{15}$ de l'AFL, mais cet espoir fut aussi déçu, en dépit du fait que l'on demandât un dollar à chaque adhérent. ${ }^{16}$

\section{c) L'ACTION POLTTIQUE}

Dès 1936, le Congrès des Organisations industrielles avait établi la Ligue non-partisane du Travail (Labor's Non-Partisan League) qui joua un rôle important dans la campagne électorale de 1936 à l'issue de laquelle Roosevelt fut réélu. Entre 1936 et 1940, des différends surgirent entre Roosevelt et John L. Lewis. La Ligue du CIO en souffrit. Il fallut attendre 1943 pour que le CIO établisse une organisation politique efficace; ce fut le Comité d'Action Politique (Political Action Committee ou PAC). Sa formation constituait un événement politique d'importance, étant due en majeure partie à Sidney Hillman qui était alors le président des «Travailleurs Réunis du Textile du CIO » (Amalgamated Clothing Workers). En 1940, Hillman avait refusé de soutenir la candidature de Wilkie (le favori de John L. Lewis) et avait au contraire réaffirmé son soutien à Roosevelt qu'i' considérait comme le meilleur ami des syndicalistes. La réélection de Roosevelt à la Présidence des Etats-Unis fut en partie due aux efforts de Hillman. Depuis lors, Roosevelt l'avait nommé chef des délégations syndicales siégeant dans les grandes commissions gouvernementales. Inquiet du vote de la loi sur les «Conflits Industriels de Guerre 》(War Labor Disputes Act) en 1943, loi qui fut votée en dépit du veto de Roosevelt, Hillman songea aux élections de 1944 qui allaient déterminer du sort des candidats du parti Démocrate en général et de Roosevelt en particulier. En tant que vétéran des combats syndicaux et très au courant de la situation qui existait aux Etats-Unis après la première guerre mondiale ${ }^{17}$, Hillman voulut s'assurer que durant la période qui suivrait la deuxième guerre, il y aurait un Président des Etats-Unis favorable au syndicalisme, à l'inverse de ce qui s'était passé en 1920 quand Harding fut élu Président. Hillman se mit donc en rapports avec Murray et le Conseil Exécutif du CIO de façon à constituer une organisation qui aiderait Roosevelt à se faire réélire. Ainsi naquit le Comité d'Action Politique.

Les méthodes du nouveau Comité suivirent de près celles des deux grands partis politiques. Il fallut d'abord effectuer un travail d'organisation par état, département, ville, arrondissement et pâté de maisons. Puis suivit le travail consistant à sonner aux portes, à solliciter les élec-

(15) A la réunion du Conseil Exécutif de l'AFL, en février 1954, on déclara officiellement que l'AFL comprenait 10,300,000 adhérents; cf. The New York Times du 2 et 5 février 1954 et AFL Convention Proceedings, 1953, p. 620 et plus haut p. 97.

(16) The New York Times, 5 février 1954.

(17) Voir Relations Industrielles, Vol. 7, no 3, pp. 309-310. 
teurs, compter les votes et distribuer les tracts. Il fallut mobiliser les parents, les associés, les amis et les convertir, et enfin utiliser toutes les ressources de la publicité moderne, la TSF, les camions à haut-parleurs, les orateurs, les réunions contradictoires, le cinéma, les tracts, les journaux, les revues, et par-dessus tout, il fa'lut coopérer avec les organisations strictement politiques travaillant dans le même but, en particulier le parti Démocrate. L'on doit admettre que Roosevelt aurait été réélu de toute façon, mais il est certain que les efforts du CIO augmentèrent le nombre de voix que Rossevelt reçut.

Aux fins d'accroître l'influence du Comité d'Action Politique du CIO, il fut constitué un Comité National des Citoyens pour lAction Politique (National Citizens' Political Action Committee ou NCPAC) avec Hillman comme président. Cette organisation avait l'avantage d'inclure des syndiqués aussi bien que des non-syndiqués. Quoique le nombre de ses adhérents fut restrejnt, la participation de gens célèbres (tels que des acteurs de cinéma) íui donna une influence hors de proportion avec le nombre de ses adhérents. En mai 1946, le PAC, le NCPAC et une nouvelle organisation dont les sympathies se rangeaient du côté syndical et du côté Roosevelt, le Comité Indépendant des Citoyens pour les Arts, Sciences et Professions (Independent Citizens' Committee of the Arts, Sciences and Professions, ou ICCASP) s'allièrent pour assurer la victoire de leurs candidats aux élections de 1946. Mais cette année-là, un grand nombre de candidats qui avaient reçu l'appui du PAC furent battus. Ceci en dépit du fait que, quelques mois avant les élections, en juin 1946, une expérience intéressante fut tentée par le NCPAC qui organisa une «Ecole de Technique d'Action Politique » à Washington. Un grand nombre d'étudiants qui assistaient à ces cours appartenaient au NCPAC, mais certains appartenaient à des organisations aussi différentes que l'Association Nationale des Industriels (National Association of Manufacturers). Les cours comprenaient «L'organisation d'un comité de citoyens », "L'utilisation du camion à haut-parleurs » et «la signification des statistiques électorales ». Au début de 1947, le NCPAC et l'ICCASP ainsi que quelques autres groupes formèrent l'organisation des Citoyens Progressistes d'Amérique (Progressive Citizens of America ou PCA). En même temps que s'établissait cette nouvelle organisation, celle des «Américains pour l'Action Démocratique» (Americans for Democratic Action ou ADA) se constituait. A l'encontre des précédentes, cette organisation n'acceptait pas les communistes. En mars 1947, le conseil exécutif du CIO recommandait aux militants du CIO de n'adhérer ni au PCA ni à l'ADA mais de donner tout leur appui au PAC. Il est à noter que le PCA mit toute son influence à la disposition d'Henry Wallace et de son parti politique, le parti Progressiste, qui était infiltré de communistes. Une des conséquences de la loi Taft-Hartley fut de convaincre le CIO qu'il devait participer de manière encore plus étroite à la vie politique du pays. 


\section{Le CIO et le problème communiste}

Un facteur contribuait de façon substantielle à fausser cette participation du CIO à la vie politique du pays aussi bien d'ailleurs qu'il contribua à son affaiblissement particulièrement après la deuxième guerre mondia'e : ce fut la pénétration communiste. Cette pénétration n'était pas nouvelle, elle remontait de fait à la formation du CIO en 1935. A cette époque, la tactique nationale communiste (suivant les ordres du Kremlin) était la politique du Front Populaire. Comme le CIO avait besoin de façon constante d'organisateurs, de nombreux communistes purent simplanter dans les nouveaux syndicats du CIO, particulièrement ceux de "lautomobile, de l'acier, du vêtement, du textile, du caoutchouc, de la construction électrique, de la fourrure et des transports. Leur influence fut à un maximum durant la deuxième guerre mondiale au moment de l'alliance militaire entre les Etats-Unis et I'URSS. Mais après 1945, la tactique communiste changea à nouveau, se conformant à la nouvelle politique du Kremlin. Certains syndicats se trouvèrent alors profondément divisés par l'action communiste qui combattait la mise en oeuvre du Plan Marshall et du Pacte Atlantique. La Ligue du parti Communiste ne coïncidait plus avec les revendications légitimes des syndicats.

Dès sa réélection en 1947 à la présidence du syndicat de lautomobile du CIO, Walter Reuther se débarassa des membres communistes et fit pression sur Philip Murray, le président du CIO, pour que celui-ci prit les mêmes dispositions à l'égard de tous les affiliés du CIO. A son congrès de 1949, le CIO expulsait les membres du Syndicat de la Construction Electrique (United Electrical Radio and Machine Workers). Ce syndicat était un des plus touchés par la propagande et l'activité communistes. Par son intermédiaire, le parti communiste américain avait la possibilité d'effectuer des actes de sabotage et d'espionnage dans les usines travaillant au compte du Ministère de la Défense. De plus, la trésorerie du syndicat, placée comme elle l'était sous le contrôle du parti communiste, donnait à celui-ci des possibilités encore plus étendues. Du point de vue syndical, les chefs du CIO n'avaient pas le choix. L'effervescence provoquée par les menées communistes au sein de ce syndicat avait atteint un tel degré qu'elle menaçait d'entraîner l'éclatement de la centrale syndicale elle-même. Le jour même de l'expulsion des membres du syndicat à tendance communiste, le CIO créa un syndicat destiné à regrouper les éléments non-communistes parmi les travailleurs de l'électricité. Ce nouveau syndicat s'attela immédiatement à la tâche de lutter contre son adversaire communiste tant sur le plan syndical que sur le plan économique et légal. Le syndicat communiste prétendait en effet garder les fonds importants recueillis chez les adhérents. Ce syndicat qui avait si souvent accusé dans le passé les tribunaux n'eut aucune hésitation à y recourir pour attaquer son adversaire.

Le CIO a contrecarré la propagande communiste aussi sur un autre front. En 1936, le CIO avait établi un « Comité Syndicaliste 
Noir, Etats-Unis 》 (Negro Labor Committee, USA) dont l'objet était d'intégrer les travailleurs noirs dans les syndicats. Jusqu'alors, les syndicats de l'AFL n'avaient accepté qu'avec difficulté les travailleurs de couleur. En 1936, quelques mois après sa formation, le CIO entreprit de recruter les ouvriers de couleur qui formaient une partie importante du personnel travaillant dans l'industrie de l'automobile. Après avoir rempli sa mission, ce comité fut plus ou moins abandonné. En 1952, le CIO décida de le rétablir pour répondre à la formation d'un Conseil Syndicaliste Noir (Negro Labor Council) par le parti communiste américain.

L'affaiblissement momentané du CIO causé par l'expulsion des communistes fut compensé par une plus grande stabilité interne. Eventuellement, plusieurs autres syndicats communistes, ceux des Travailleurs de l'Alimentation et du Tabac, des Employés de Bureau et des Employés d'Administration Publique, des Télégraphes et des Téléphones, des Fourreurs, des Cuisiniers et Stewarts de Navires et des Pêcheurs furent expulsés du CIO. Cette épuration élimina pratiquement l'influence communiste au sein du mouvement ouvrier et eut aussi pour résultat de rapprocher le CIO de l'AFL où le problème communiste n'existait pour ainsi dire pas. La question communiste ne constitua done pas d'obstacle à la fusion de l'AFL et du CIO.

\section{d) L'ACTION POLITIQUE DE L'AFL-CIO}

Depuis 'a fusion de l'AFL et du CIO en 1955, la Ligue du Travail pour l'instruction politique et le Comité d'Action Politique se sont groupés dans le Comité pour l'Education Politique (Committee on Political Education ou COPE). Ce Comité poursuit deux buts :

1) L'éducation politique des ouvriers dont beaucoup ne votent pas, une de ses tâches essentielles est donc de les inciter à voter, et;

2) L'obtention d'engagements favorables au «Labor» de la part des candidats aux élections en échange en quelque sorte des votes ouvriers, une sorte de négociation de conventions collectives sur le plan politique.

Le mouvement syndical se considère aujourd'hui «forcé» d'entreprendre des activités politiques :

\&...parce que les ennemis du mouvement syndical ont prouve qu'ils peuvent, par l'intermédiaire de la politique, affaiblir de façon sérieuse nos efforts tendant à obtenir un meilleur standard de vie pour nos adhérents et pour notre pays. \$*

De 1946 à 1957, 10,200,000 exemplaires des façons de voter de chaque député (représentative) concernant 19 questions d'importance

(*) Rapport du Conseil Exécutif au Congrès de l'AFL-CIO en 1957 cité par l'AFL-CIO News, 7 décembre 1957, p. 13. 
majeure et 20 questions d'importance pour chaque sénateur furent imprimés et distribués aux adhérents par la poste. Il n'est pas certain d'ailleurs que cet énorme travail atteigne les résultats désirés. L'AFLCIO avait soutenu officiellement en 1956 Adlai Stevenson et Estes Kefauver contre Eisenhower et Nixon, mais ce furent les candidats républicains qui furent élus. De plus :

* Il n'y eut pas de changement important dans le nombre d'amis ou d'adversaires du mouvement syndical (à la Chambre des Députés) « House of Representatives 》, étant donné que nous avons perdu à peu près le même nombre d'amis que nous avons gagné. \$*

Et ce fut aussi le cas pour le Sénat. Pour les élections locales :

- Il est à noter que les gains les plus importants furent acquis dans les états où les fédérations de l'AFL et du CIO sont fusionnées. >*

Immédiatement après les élections présidentielles de 1956, COPE se mit à l'oeuvre pour obtenir de meilleurs résultats à l'occasion des élections municipales, les élections d'état et les élections spéciales qui ont lieu pour remplacer un «representative » ou un sénateur décédé ou qui a démissionné.

\& Dans ces (élections), la force de notre organisation, pas aussi apparente dans une élection de la grandeur et de la portée d'une élection présidentielle, devint évidente. \$*

Dans le Wisconsin, le Texas, le New Jersey et d'autres états, les candidats soutenus par le «Labor » furent élus. On découvrit en 1956 que le pourcentage de syndiqués qui avaient fait les démarches nécessaires pour pouvoir voter variait suivant les quartiers de 23 à $45 \%$ à Indianapolis*, ce qui donne une idée pour le reste du pays.

Le pourcentage semble d'ailleurs plus bas pour les syndicats du sud. En 1956, sous l'égide de COPE, 30,360,000 tracts, pamphlets ou livres furent distribués, y compris un manuel de 256 pages ayant trait à l’organisation politique et un «manuel de conférencier de 374 pages ». *

Du 5 décembre 1955 au 30 juin 1957, le Comité d'Education Politique (COPE) de l'AFL-CIO a dépensé plus de 718,000 dollars, soit environ plus de 350 millions de francs. * Ces sommes, bien entendu,

(*) Ibid., p. 312 .

(*) Report of the Executive Council of the AFL-CIO, Second Convention, Atlantic City, New Jersey, December 5, 1957, American Federation of Labor and Congress of Industrial Organizations, p. 111.

(*) Ibid., p. 112.
(*) Ibid., p. 113.
(*) Ibid., p. 114.
(*) 
servent à financer l'utilisation de camions à haut-parleur, l'impression de tracts, etc. Il faut souligner le rôle des volontaires et des militants dans la campagne électorale. Sans eux, sans leur grand nombre, le but ne serait pas atteint.

Le 16 août 1956, (le) secrétaire-trésorier Schnitzler est apparu devant le comité... du parti Démocrate et a présenté les vues de notre organisation ayant trait aux problèmes les plus importants de notre pays. Le 16 aoôt 1956, (le) secrétaire-trésorier Schnitzler est apparu devant le comité... du parti Républicain et a fait une présentation similaire. $>*$

\section{Pendant les élections présidentielles de 1956 :}

« Votre COPE a préparé et distribué à tous les affiliés (de l'AFLCIO) une comparaison détaillée des plate-formes Républicaine et Démocrate avec les propositions placées devant ces organisations par (le) Président Meany et le Secrétaire-trésorier Schnitzler. Il (COPE) a aidé les candidats soutenus (par l'AFL-CIO) à organiser des réunions avec les représentants de nos organisations. Il a préparé et distribué des pamphlets à l'usage de nos adhérents décrivant de façon brève les activités des candidats pour la présidence et la vice-présidence... Mais les plus gros efforts de COPE se dirigeaient vers les deux (objectifs) fondamentaux de l'éducation politique syndicale; des citoyens informés et immatriculés pour voter. \$*

Suivant les efforts du mouvement syndical, le nombre de syndiqués qui s'immatriculèrent pour voter monta de 5 à $6 \%$. $^{*}$

- Notre tâche fondamentale consiste à faire voter chaque militant, ainsi que chaque membre adulte de sa famille... Egalement important est le devoir d'être informé... Nous considérons la distribution des listes montrant la manière dont les candidats ont voté sur des sujets d'importance comme un pas essentiel dans cette direction... il est vital que le mouvement syndical américain se trouve en position d'aider financièrement les candidats dont les vues et les activités pour la justice sociale les empêchent de recevoir des contributions d'autres sources. Nous croyons que cette aide est juste et dans l'intérêt public et nous avons donc fait campagne chaque année pour obtenir des contributions volontaires de nos adhérents dans ce but, * $^{*}$

Le rapport du Conseil Exécutif au Congrès de l'AFL-CIO en 1957 déclarait qu'il n'était pas nécessaire de faire une analyse détaillée des

(*) Second Constitutional Convention of the American Federation of Labor and Congress of Industrial Organizations, 1957 Proceedings, Atlantic City, New Jersey, December 11, 1957, Report of Fifth Day - Wednesday Morning Session, p. 23.

(*) Report of the Executive Council of the AFL-CIO, Second Convention, Atlantic City, New Jersey, December 5, p. 105.

(*) Ibid. p. 108.

(*) Ibid., p. 109. 
répercussions sérieuses de la loi Taft-Hartley parce que les dénonciations du Congrès de 1955 à son égard :

«...sont aujourd'hui aussi valides qu'elles l'étaient à ce moment-là...

De plus, les décisions du NLRB (qui est chargé d'appliquer la loí)

sont telles qu'elles renforcent encore les clauses anti-syndicales de

la loi. > $^{*}$

Le congrès de l'AFL-CIO en 1957 passa une résolution tendant à accroître le nombre de femmes utilisées par les affiliés dans le cadre du programme d'éducation politique.

En 1958, COPE a étendu le champ de ses activités et plus particulièrement le renforcement des liens qui unissent les groupes minoritaires aux Etats-Unis au mouvement syndical.

c...les patrons maintenant reconnaissent généralement, sans désaccord sérieux la justice d'augmentations de salaires de façon à compenser l'augmentation du coût de la vie et aussi pour faire progresser quelque peu le standard de vie des travailleurs. $>$ *

\section{e) LA QUESTION dE LA FORMATION D'UN PARTI POUTTQUe \\ A BASE SYNDICALE}

En raison de la manière « indirecte » de procéder du mouvement syndical en matières politiques, la question a été posée depuis un grand nombre d'années de savoir si celui-ci n'aurait pas intérêt à établir son propre parti politique. La première réponse à cette question importante était, qu'un parti politique basé sur les syndicats était nécessairement fonction de lunité organique du mouvement. Un mouvement syndical divisé ne pouvait donner naissance à un parti politique unifié. Mais cette considération, pour importante qu'elle fût, n'était pas la seule. Un grand pourcentage de syndiqués, impossible à déterminer de façon précise, est inscrit au parti Démocrate ou Républicain. Qu'un nouveau parti politique soutenu par leur syndicat surgisse, ces syndiqués ne changeraient pas nécessairement leur affiliation politique. Il faudrait donc envisager un grand nombre de difficultés pour faire fonctionner un parti qu'un nombre plus ou moins grand de syndiqués se refuserait à soutenir. Un conflit de «loyauté » créerait des controverses et des dissensions au sein des syndicats et du mouvement ouvrier en général.

D'autre part, un parti politique ouvrier aurait à affronter une opposition considérable, non seulement de la part des deux grands partis depuis longtemps en existence, mais de par son programme même. Il est en effet normal de penser que la plate-forme politique d'un tel parti

(*) Rapport du Conseil Exécutif au congrès de l'AFL-CIO en 1957, cité par l'AFL-CIO News, 7 décembre 1957, p. 13.

(*) Report of the Executive Council of the AFL-CIO, Second Convention, Atlantic City, New Jersey, December 5, 1957, American Federation of Labor and Congress of Industrial Organizations, pp. 141-142. 
serait plus \& radicale» que celle des deux autres et serait donc combattue avec plus de vigueur par eux et par les forces conservatrices du pays. A ses difficultés d'ordre intérieur, le nouveau parti aurait donc à ajouter des obstacles extérieurs d'importance. De plus, le grand nombre de candidats que le nouveau parti aurait à présenter serait tiré en majeure partie des cadres syndicaux, les appauvrissant d'autant. L'établissement d'un nouveau parti nécessiterait la dépense de vastes sommes d'argent que les syndicats peuvent peut-être fournir mais au risque de s'amoindrir financièrement et de ne pas être en mesure d'endosser les difficultés de trésorerie généralement causées par les grèves. Il est difficile de comparer les difficultés soulevées par la formation d'un nouveau parti aux profits que les syndicats pourraient en tirer. Il est aussi difficile de conclure que la simple formation d'un nouveau parti inciterait les chefs des deux autres à faire de plus grandes concessions au mouvement ouvrier. Les partis venant en troisième lieu qui ont fait leur apparition au cours de l'histoire des Etats-Unis ont tous échoué. Les deux grands partis américains groupent, c'est-à-dire unissent des gens appartenant à toutes les classes économiques et sociales. Pour bon nombre d'Américains, la formation d'un parti axé exclusivement sur la défense des intérêts ouvriers représenterait une menace considérable posant de graves problèmes politiques. Finalement, et c'est une des questions essentielles: dans quelle mesure un parti ouvrier peut-il exister en l'absence d'un esprit de classe? $\mathrm{CIO}$ :

Comme George Meany le déclara au congrès constitutif de l'AFL-

« Je suis assez amusé par toute cette agitation concernant l'activité politique du mouvement syndical, concernant le contrôle des votes par les chefs syndicaux. Je suis certain qu'ils (nos détracteurs) savent que nous ne contrôlons pas les votes. Personne ne peut dire au travailleur américain comment il doit voter... Ils (nos détracteurs) se font du souci au sujet d'un parti travailliste. Eh bien! je ne suis conscient d'aucun sentiment favorisant (la formation d')un parti travailliste, et je ne suis conscient d'aucun sentiment favorisant le contrôle d'un des partis politiques par le mouvement syndical... Le challenge est lancé dans les chambres législatives, et notre réponse est l'éducation politique et l'activité politique. »*

A l'heure actuelle, la formation d'un parti politique basé sur les syndicats est donc peu probable. Par contre, il est possible que l'influence actuelle de la grande centrale syndicale aille en augmentant sur les deux partis politiques. Mais cette influence sera toujours fonction de la puissance des effectifs syndicaux, et de l'attitude du gouvernement. Il est indéniable que la formation d'un troisième parti représentatif du mouvement syndical serait favorisée par certains éléments de l'AFL-

(*) 1955 Constitutional Convention Proceedings, New York, N.Y., December 5-8, 1955, American Federation of Labor and Congress of Industrial Organizations, pp. 27-28. 
CIO, mais ces èléments sont généralement en infime minorité. Il s'en trouve peut-être un peu plus parmi l'ancienne faction CIO qui, en raison de ses origines, a toujours été plus favorable à l'action politique pure et simple et à une plus grande intervention gouvernementale pour améliorer les conditions de travail que l'AFL. De paire avec cette intervention gouvernementale qui intéresse le mouvement syndical, il existe une intervention syndicale intéressant le gouvernement.

\title{
f) Participation du Mouvement syndical aU GoUvernement
}

\begin{abstract}
« Mais cette union, le « Congrès des Organisations Industrielles 》, est destinée à vivre, à prospérer, à grandir en force et en influence, non seulement dans les affaires de l'industrie et de la finance, mais aussi bien dans les affaires du gouvernement. ${ }^{18}$
\end{abstract}

Quoique cette déclaration de la part d'un chef syndical soit récente, le fait pour le mouvement syndical remonte à 1913, lors de l'établissement d'un Ministère du Travail distinct du Ministère du Commerce. Le personnel de ce Ministère ne comprenait jusqu'au début de la deuxième guerre mondiale qu'un nombre restreint d'anciens militants syndicalistes. Durant la première guerre mondiale, le Président des Etats-Unis, Wilson, avait nommé le président de l'AFL, Gompers, à d'importantes commissions gouvernementales. Durant la deuxième guerre mondiale, le Président Roosevelt désigna un grand nombre de militants de l'AFL et du CIO à des postes importants dans presque toutes les branches du gouvernement. Il estimait que la situation demandait la coopération complète du mouvement syndical. ${ }^{19}$

A la fin de la guerre, en plus diun accroissement considérable du nombre de ses adhérents, le mouvement syndical avait acquis une position sans précédent au sein de la société américaine. Certaines universités décernèrent des diplômes «honoris causa 》 à des chefs syndicalistes. ${ }^{20}$ Le Ministère des Affaires Etrangères (Department of State) augmenta le nombre de ses attachés ayant pour mission d'étudier les questions ouvrières à l'étranger (Labor Attachés). Mais un des exemples les plus frappants de changement du statut syndical fut l'établissement d'une Division du Travail au sein de l'Organisation pour la Coopération Economique (Economic Cooperation Administration ou ECA, plus connue sous le nom de Plan Marshall) qui fut établie en 1947. Cette Division du Travail fut établie à la requête du premier directeur du Plan Marshall, Paul Hoffman. Ce dernier signa un accord avec William Green, président de l'AFL et Philip Murray, président du CIO, aux termes duquel une représentation égale de militants de l'AFL et du CIO serait donnée à cette Division. De manière concrète, on tenait compte du point de vue syndical en matière de politique

(18) CIO Convention Proceedings, 1952, p. 232.

(19) Voir Relations Industrielles, Vol. 17, no 3, pp. 317-318.

(20) Phil Murray, Président du CIO et James Carey, Secrétaire-trésorier du CIO furent honorés de cette manière en 1948. 
étrangère. L'AFL et le CIO avaient donné tout leur appui au Plan Marshall dès ses débuts, ainsi qu'à l'Organisation du Point IV, chargée du développement des pays «arriérés» (underdevelopped) au point de vue économique.

Cette position du mouvement syndical au sein de la société américaine, position nouvelle appuyée et «sanctionnée " par les hautes fonctions accordées aux militants syndicalistes, ne signifiait pas qu'un accord complet existait entre le gouvernement et les deux grandes centrales syndicales. Si le syndicalisme américain est foncièrement anticommuniste et s'il est en accord général avec la politique extérieure du gouvernement, compte tenu de certaines exceptions, ${ }^{21}$ en matière de politique intérieure, il existe de nombreuses divergences entre le gouvernement et les syndicats. Ces divergences soulignent d'ailleurs l'indépendance du «Labor 》, sur toutes les questions, quelles qu'elles soient, et se reflètent aussi dans la représentation syndicale au sein du gouvernement. Jusqu'à présent, l'AFL-CIO n'a pas montré beaucoup de désir à accroître de quelque manière que ce soit sa participation au gouvernement. Il semblerait même que la tendance opposée soit le cas.

\section{g) Polttique étrangère}

Dans la poursuite de leur politique étrangère, les deux grandes centrales syndicales ont envoyé leurs représentants dans presque tous les pays du monde libre pour aider moralement et financièrement les syndicats se battant contre l'emprise communiste. Un examen du compte-rendu du congrès de l'AFL de 1952 relate une discussion de la situation syndicale et politique dans les pays suivants : L'Afrique du Sud, l'Alaska, l'Algérie, l'Allemagne, l'Angleterre, l'Argentine, l'Australie, I'Autriche, la Bolivie, le Brésil, la Bulgarie, le Canada, la Chine, la Corée, la République Dominicaine, l'Egypte, l'Equateur, l'Espagne, la Finlande, l'Ile de Formose, la France, la Grèce, le Guatémala, Haïti, Hawaii, les Indes, l'Indo-Chine, l'Indonésie, l'Iran, l'Irlande, Israël, l'Italie, le Japon, la Lybie, le Maroc, le Mexique, le Pakistan, le Pérou, Puerto-Rico, la Tchécoslovaquie, Terre-Neuve, la Tunisie, la Turquie, I'URSS, I'Uruguay, le Vénézuéla et la Yougoslavie. A son congrès de 1952, l'AFL entendit les rapports des représentants qu'elle avait envoyés dans un certain nombre de pays mentionnés ci-dessus, quoique, pour certains pays, l'exposé se bornât au rapport du Conseil Exécutif de l'AFL, rapport qui fut adopté au congrès. Par l'intermédiaire de ce rapport et de ses résolutions, l'AFL faisait connaître au public américain et au gouvernement sa position officielle en matière de politique étrangère. En sa qualité de membre de la Confédération Internationale des Syndicats Libres qu'elle aida à fonder en 1949, de concert avec les syndicats britanniques et d'autres syndicats pour lutter contre la Fédération Syndicale Mondiale qui était devenue une succursale soviétique

(21) Par exemple, l'AFL prit position contre les négociations avec l'Espagne. 
et pour entreprendre une action syndicale internationale efficace, Y'AFL avait pris sa part de responsabilité dans la formulation d'une politique syndicale mondiale.

Le CIO, lui aussi, s'intéressait de façon directe à la politique étrangère du gouvernement et désirait voir des militants syndicalistes participer activement à la formulation d'une telle politique dans les sphères gouvernementales. Témoin cette déclaration faite au congrès du CIO en 1952 :

«Le CIO réitère l'assurance que notre pays bénéficiera directement de la nomination d'hommes et de femmes qui ont fait leur apprentissage dans les rangs ouvriers à des situations importantes dans les divisions du schéma de la politique et des opérations du Ministère ies Affaires Etrangères... Le mouvement ouvrier américain a des possibilités de communications et de relations à l'étranger dont l'utilisation est essentielle à la poursuite heureuse d'une politique étrangère démocratique... La participation ouvrière à l'ECA est responsable pour beaucoup du succès du programme de réhabilitation. , 22

Quoique les activités internationales du CIO fussent moins considérables que celles de l'AFL, le CIO avait aussi un représentant en Europe et s'intéressait aux affaires internationales tant par le rôle qu'il a joué au sein de la Confédération Internationale des Syndicats Libres (CISL) que par le soutien qu'il a donné aux activités de l'Organisation des Nations Unies. L'AFL-CIO maintient un représentant en Europe et continue à participer activement aux affaires de la CISL. L'intérêt que l'AFL-CIO prend aux affaires internationales se reflète dans le nombre et la diversité des délégués étrangers à ses conventions.

Sans compter les messages d'amitié qui affluèrent de toutes les parties du monde, on peut se rendre compte de l'intérêt international que présente le mouvement syndical en jetant un coup d'oeil sur la liste des délégués étrangers; ils vinrent du Japon, d'Angleterre, de la Malaisie, du Liban, du Canada, de l'Amérique du Sud, d'Allemagne, d'Italie, du Brésil, du Maroc et du Kenya.

De plus l'AFL-CIO participa de façon active à la quatrième conférance du Comité Unifié Syndical Mexico-Etats-Unis (Joint United Stater-Mexico Trade Union Program) qui se réunit au Mexique en 19:7. Les résolutions de la conférence demandaient entre autres, des salaires minima pour les travailleurs mexicains et l'organisation de syndicats pour les mêmes travailleurs.

\section{h) Politique INTÉRIEURE}

En ce qui concerne les questions de politique intérieure, il est difficile de trouver un secteur de l'économie américaine dans lequel ne (22) CIO Convention Proceedings, 1952, Resolution No. 16, Foreign Policy, p. 380 . 
se fasse pas sentir l'influence du mouvement ouvrier. Cette influence se montre non seulement à l'intérieur des Etats-Unis mais dans les nouveaux Etats comme l'Alaska et Hawaii. Etant données l'influence et la prépondérance du gouvernement fédéral dans de nombreux secteurs de l'économie, les décisions qui affectent de façon vitale cette économie sont prises par le Congrès fédéral. C'est donc à Washington que l'influence syndicale se fait le plus sentir. Pendant longtemps et encore aujourd'hui les efforts de l'AFL-CIO des associations des employés de chemin de fer (Railroad Brotherhoods) et de tous les syndicats en général furent dirigés contre la loi Taft-Hartley. Jusqu'à présent ces efforts sont restés sans succès. Mais en dehors de ces activités dirigées contre une loi que les syndicats considèrent comme anti-ouvrière, il faut souligner que le mouvement ouvrier représente une force politique positive.

\section{1. $A F L$}

L'AFL demanda au Congrès fédéral d'exempter de taxes toutes les pensions et retraites, d'augmenter les fonds qui permettent au Ministère du Travail de fonctionner, d'autoriser les états à accorder des allocations aux femmes enceintes et aux enfants de soldats. En même temps, lAFL se félicita du passage de l'Amendement Benton à l'Acte de Sécurité Mutuelle de 1951, ${ }^{28}$ ledit amendement déclarant que l'aide à l'étranger doit être administrée de façon à :

< Encourager, là où il est possible de le faire, le développement et le renforcement des syndicats libres dans la mesure où ceux-ci sont chargés de la négociation des contrats collectifs dans ces pays. $\$ 24$

Au cours de 1952, l'AFL s'efforça de faire échouer certains projets de loi qui auraient eu pour effet d'abaisser l'âge minimum légal pour la main-d'oeuvre enfantine, particulièrement dans les états du Kentucky, de New-York et du Maryland. ${ }^{25}$ L'AFL demanda aussi au Congrès fédéral d'augmenter les sommes destinées au Bureau des Enfants de l'Agence de Sécurité Fédérale (Federal Security Agency), ce qui fut fait. L'AFL continua sa lutte pour les droits civils et, en 1952, trente-cinq projets de loi les concernant furent soumis au Congrès fédéral, mais aucun de ces projets ne fut voté. Avec succès, l'AFL s'est battue pour une augmentation des pensions des fonctionnaires. ${ }^{28}$ Une résolution de l'AFL demandait la simplification des formalités douanières, tandis qu'une autre s'élevait contre l'importation de poissons et de crustacés. ${ }^{27}$ L'AFL s'opposa au placement de commandes gouvernementales dans les usines où les travailleurs ne sont pas syndiqués. Elle s'intéressa de très près aux amendements à la loi de la Production

(23) Concernant l'Agence de Sécurité Mutuelle qui a remplacé l'ECA et qui fut elle-même remplacée par l'Agence de coopération internationale (ICA).

(24) AFL Convention Proceedings, 1952, p. 101.

(25) Ibid., p. 283.

(26) Ibid., p. 244.

(27) Ibid,, pp. 273 et 494. 
pour la Défense (Defence Production Act), particulièrement en ce qui concerne la stabilisation des salaires et la lutte contre l'inflation. Les propositions que l'AFL fit à ce sujet ne furent pas acceptées « in toto » et le rapport du Conseil Exécutif de l'AFL signala le danger inflationniste qui existait dans la loi. L'AFL eut plus de succès en ce qui concerne ses recommandations au Congrès fédéral à l'égard de la maind'oeuvre civile et à la mobilisation d'hommes appartenant à certains métiers. ${ }^{28}$ L'AFL soumit des recommandations au Congrès fédéral pour que les prêts du gouvernement fédéral aux régions inondées soient transformés en dons purs et simples, pour la lutte contre la discrimination raciale et l'augmentation du nombre d'immigrants aux Etats-Unis. A noter que cette dernière demande est assez nouvelle de la part du syndicalisme américain. Jusqu'à une période aussi récente que celle qui suivit la première guerre mondiale, les syndicats étaient généralement opposés à l'immigration. ${ }^{29}$ Les recommandations de l'AFL au Congrès fédéral contenaient aussi une demande de réduction des taxes sur l'alcool, de législation plus sévère régissant la vente des narcotiques, de soutien de l'UNESCO, de soutien des écoles et lycées, d'aide de la part du gouvernement fédéral en vue d'accroître les émoluments des professeurs, de réserver certaines émissions télévisées à l'éducation, d'établissement d'une Commission de Pratiques d'Emploi Equitables (Fair Employment Practices Commission ou FEPC) fédérale rendant illégale toute discrimination dans l'embauchage qui serait basée sur la nationalité, la couleur ou la race de l'employé et une défense des hôpitaux réservés aux marins. ${ }^{30}$

Dans les demandes que l'AFL avait faites au parti Républicain et au parti Démocrate, l'élimination de la loi Taft-Hartley prenait la première place. Venaient ensuite la lutte contre l'inflation, le contrôle des loyers, la construction de logements, les assurances sociales, la médecine sociale, l'aide du gouvernement fédéral aux écoles, un examen des droits civils et la poursuite d'une politique étrangère basée sur la lutte contre le communisme. ${ }^{31}$

\section{2. $\mathrm{CIO}$}

Pour considérable qu'il soit, le rôle du CIO n'avait pas acquis l'ampleur de ce'ui de l'AFL en matière de politique intérieure. Le CIO ne possédait pas les effectifs considérables de l'AFL et par suite n'avait pas à sa disposition les mêmes moyens financiers. Le CIO ne déclarait pas officiellement le chiffre de ses adhérents, mais on les estimait à environ 5,000,000 en 1953. ${ }^{32}$ Le CIO avait une conception du rôle du gouvernement différente de celle de l'AFL. Alors que pour l'AFL le

(28) Ibid., p. 257.

(29) Ibid., p. 147; cf. aussi Relations Industrielles, Vol. 17, no 3, p. 306.

(30) Ibid., pp. 26 et 462.

(31) Ibid., pp. 94 et 98.

(32) The World Almanac and Book of Facts for 1954, N.Y. World Tel., New

York, 1954, p. 80 . 
rôle du gouvernement dans l'économie devait se faire sentir surtout dans certains secteurs, pour le CIO, le rôle du gouvernement était beaucoup plus vaste. En effet, la conception du CIO du rôle du gouvernement dans la vie du pays était en accord avec les conceptions du New Deal. Il ne faut pas oublier que la naissance du CIO se fit à une époque où l'intervention du gouvernement fédéral dans les affaires internes du pays fut massive et en quelque sorte révolutionnaire. Les conceptions du CIO ont été fortement influencées par cette période. Le CIO concevait donc une intervention plus prolongée et plus étendue du gouvernement fédéral dans le secteur économique et en général dans la vie du pays. Le CIO réclamait aussi plus de «p'anisme» de la part du gouvernement que l'AFL. D'où l'importance que le CIO attachait à ses activités strictement politiques et le fait que le CIO ait été la première des deux grandes centrales syndicales à former un Comité d'Action Politique. C'est pourquoi aussi le compte-rendu du congrès du CIO de décembre 1952 donnait une liste détaillée avec commentaires des élections présidentielles de novembre 1952. ${ }^{33}$ Le CIO accordait beaucoup d'importance à, ce qu'il appelait, l'éducation politique. Avec le concours de la télévision, d'émissions radiophoniques et de tracts. ${ }^{34}$ Le CIO essayait de donner aux électeurs et particulièrement à ses adhérents une idée précise des problèmes politiques et des candidats.

En ce qui concerne 'es demandes que le CIO faisait au Congrès fédéral, un grand nombre était similaire à celles de l'AFL. Mais alors que l'AFL se préoccupait surtout des monopoles internationaux et des cartels, ${ }^{35}$ le CIO se préoccupait plus des monopoles américains et attaquait le Congrès pour ne pas avoir défendu les petites entreprises contre l'emprise monopoliste. ${ }^{36}$

\section{L'AFL-CIO}

La po'itique intérieure de l'AFL-CIO est, à l'heure actuelle, empreinte des conceptions des deux anciennes confédérations, conceptions qui ne présentaient vraiment pas de différences fondamentales. L'amalgamation du personnel employé par les deux organisations semble se fondre elle aussi en une fusion des idées avec cependant une tendance à l'ancienne orientation AFL. Il n'y a cependant pas de solution de continuité ainsi que nous l'avons déjà vu.

\section{f) Pragmatisme syndical}

Lorsque l'on passe en revue l'activité politique des syndicats américains, on ne manque pas d'être frappé par l'absence de doctrine et d'idéologie. Le mouvement syndical américain est en effet caractérisé

(33) CIO Convention Proceedings, 1952, pp. 424-432.

(34) Quinze millions de tracts furent distribués par le PAC en 1952; cf. CIO Convention Proceedings, 1952, p. 186.

(35) AFL Concention Proceedings, 1952, p. 147.

(36) CIO Convention Proceedings, 1952, p. 141. 
par un pragmatisme renforcé par les leçons de multiples expériences. L'activité politique du mouvement tend donc à un changement graduel à l'intérieur du système et non pas à un changement fondamental du système lui-même. Le mouvement syndical accepte le milieu dans lequel il se trouve et ne songe qu'à améliorer son modus vivendi, sans plan d longue échéance. Ce fait ressort particulièrement à la lumière de l'illégalité des syndicats au début du 19ème siècle. D'ailleurs, même à cette époque, les syndicats n'étaient pas révolutionnaires, ce qui est une démonstration supplémentaire de leur caractère fondamentalement « évolutionniste \$. Plus récemment, en 1934 et en 1935, l'AFL refusa de voter en faveur d'une résolution préconisant la grève générale et cela pendant une période qui a été qualifiée de révolutionnaire dans l'histoire des Etats-Unis. ${ }^{37}$

Il est probable que l'activité politique des syndicats ira en augmentant en fonction de l'importance grandissante et des besoins croissants de leurs effectifs. L'efficacité et l'intensité de cette activité dépendront en grande mesure de la solidarité du mouvement syndical maintenant que l'unité du mouvement est faite et de la pression légale dont il sera lobjet. On peut remarquer à ce sujet que la loi Taft-Hartley avait beaucoup fait pour rapprocher les éléments syndicaux. Le fait de participer à la même lutte avait renforcé le sentiment de solidarité qui existait déjà. Mais la puissance politique que recherchent les syndicats n'est pas un objectif «per se». Les syndicats cherchent à augmenter leur puissance politique de façon à assurer par une législation appropriée leurs objectifs économiques. Ceux-ci en général sont destinés à augmenter le bien-être des syndiqués, et par conséquent, dans l'esprit des chefs des syndicats, celui du pays. L'AFL-CIO a une division de législation qui s'occupe surtout, en collaboration avec les autres divisions du travail de représentation, des vues syndicales au Congrès. C'est ainsi qu'entre 1955 et 1957, les représentants législatifs de PAFL-CIO ont témoigné 115 fois devant des comités ou sous-comités du Congrès. * La ligne de conduite de l'AFL-CIO en matière de législation se trouve déterminée par le congrès de l'AFL-CIO. Entre les congrès, c'est le Conseil Exécutif de I'AFL-CIO qui s'en charge.

En une occasion particulière, George Meany précisait le rôle du mouvement ouvrier américain de la façon suivante:

- Nous devons avoir un instrument assez fort pour maintenir les niveaux (de vie) des travailleurs de ce pays, pour les protéger contre les catastrophes par l'intermédiaire d'un (système) d'assurances sociales efficace et par dessus tout pour sauvegarder leurs libertés élémentaires... C'est dans ce but... (que) nous comptons utiliser tous les moyens qui sont légalement à notre portée en tant que citoyens.

(37) Cf. The Twenty-Year Revolution, Chesly Manly, Henry Regnery Co., Chicago, 1954.

(*) Report of the Executive Council of the Second Convention, etc., p. 503. 
Sans aucun doute, ces moyens comprendront l'action politique non seulement pour défendre notre mouvement contre une législation destinée à nous détruire mais aussi pour améliorer encore davantage le niveau de vie américain... La scène des batailles ne se trouve plus maintenant dans les usines ou à leurs portes. Elle a été transférée à l'intérieur des chambres législatives du Congrès et dans les législatures des Etats... $>38$

(38) Discours de George Meany, le 4 novembre 1955, à l'inauguration du noubel immeuble du Syndicat International des Chauffeurs.

\section{LA REVUE DE L'ACTION POPULAIRE} Reoue mensuelle publiée par les Jésuites de l'Action Populaire de Paris

Réduire le temps de travail?

Sommaire du numéro 155, février 1962

La recherche historique

R.-H. Guerrand

Une réflexion philosophique

E. Rmenu

L'analyse économique

Fr. SELLIER

Sociologues et organisateurs

J. Dubors

J. Piel-Desruisseaux

J. Hallatre

Options et perspectives

A. Demonchaux

A. Le RoY

J. Charpentreau

\section{Le droit à la paresse .}

Homme-au-travail, homme-au-loisir

La durée du travail dans l'économie

Une vie de travail: cycles et durée

En agriculture, rythmes et temps de travail

Migrations alternantes des travailleurs

Les revendications syndicales

Comparaisons internationales

Scandaleux loisir

Abonnement annuel: \$5.50, Periodica, 5090, Ave Papineau, Montréal 34.

Rédaction: 15, rue Marcheron, Vannes (Seine). 